\title{
Neurofibrosarcoma of the radial nerve in von Recklinghausen's disease with metastatic angiosarcoma
}

\author{
R. A. A . M A C A U L A Y \\ From the Department of Pathology (Neuropathology), Stanford University School of Medicine, \\ Stanford, California, USA
}

SUMMARY A case of von Recklinghausen's disease with multiple neurofibromatosis is described in which death was caused by intracerebral haemorrhage due to metastatic angiosarcoma. The primary site was a peripheral nerve tumour which, on microscopic examination, showed both angiosarcoma and neurofibrosarcoma with transitional zones between the two. This is interpreted as another example of divergent differentiation in malignant nerve sheath tumours, and the case is compared with the two similar examples found in the literature.

The development of malignancy in a peripheral nerve tumour of von Recklinghausen's disease is well-known (Harkin and Reed, 1969; Krucke, 1974; Russell and Rubinstein, 1977). Distinction between typical forms of malignant Schwannoma, malignant neurofibroma or, alternatively, neurofibrosarcoma is sometimes possible on morphological grounds but is often difficult to establish in their most undifferentiated forms (Krucke, 1974).

A rare but well-established feature in the malignant tumours is the presence of focal metaplastic change into other mesenchymal elements such as cartilage, osieoid, bone, and skeletal muscle (Russell and Rubinstein, 1977).

This paper presents in detail the development of yet another example of divergent differentation in malignant neurofibrosarcoma originating in von Recklinghausen's disease, namely that of angiosarcomatous features.

\section{Case report}

The patient was an 18 year old white male with von Recklinghausen's disease diagnosed on the basis of numerous café-au-lait spots over his trunk and limbs, and multiple neurofibromas. He first

Address for correspondence and reprint requests: R. A. A. Macaulay, Department of Forensic Medicine, Edinburgh University Medical School, Teviot Place, Edinburgh, Scotland.

Accepted 14 December 1977 presented with a right axillary mass which had been enlarging for six months, right wrist drop, haemoptysis, and a $9 \mathrm{~kg}$ weight loss. Chest radiography showed diffuse right upper lobe infiltration. Biopsy of the axillary mass demonstrated a neurofibrosarcoma. He was presumed to have pulmonary metastases, and received radiotherapy to the right lung, mediastinum, and the axillary mass. Two months later he was readmitted complaining of headaches, nausea, and vomiting. He was disorientated and had papilloedema. Brain scan $\left({ }^{99^{\mathrm{m}}} \mathrm{Tc}\right)$ and electroencephalography demonstrated bilateral frontal lobe lesions. He died soon after admission.

\section{NECROPSY FINDINGS (OG-160)}

The right radial nerve $10 \mathrm{~mm}$ distal to its origin in the brachial plexus, expanded into a firm pale fusiform mass approximately $150 \times 50 \times 50 \mathrm{~mm}$, which compressed and was firmly attached to the ulnar nerve (Fig. 1). The mass was easily separated from the surrounding musculature and from the sheath of the axillary artery and vein. On section it was firm, white, and whorled with a haematoma in the region of a wedge shaped excisional biopsy, and with several other areas of haemorrhage distant from the biopsy site.

Both lungs contained round, grey-white haemorrhagic parenchymatous and subpleural nodules. None was associated with any of the major bronchi. The nodules measured up to $15 \mathrm{~mm}$ in diameter. 


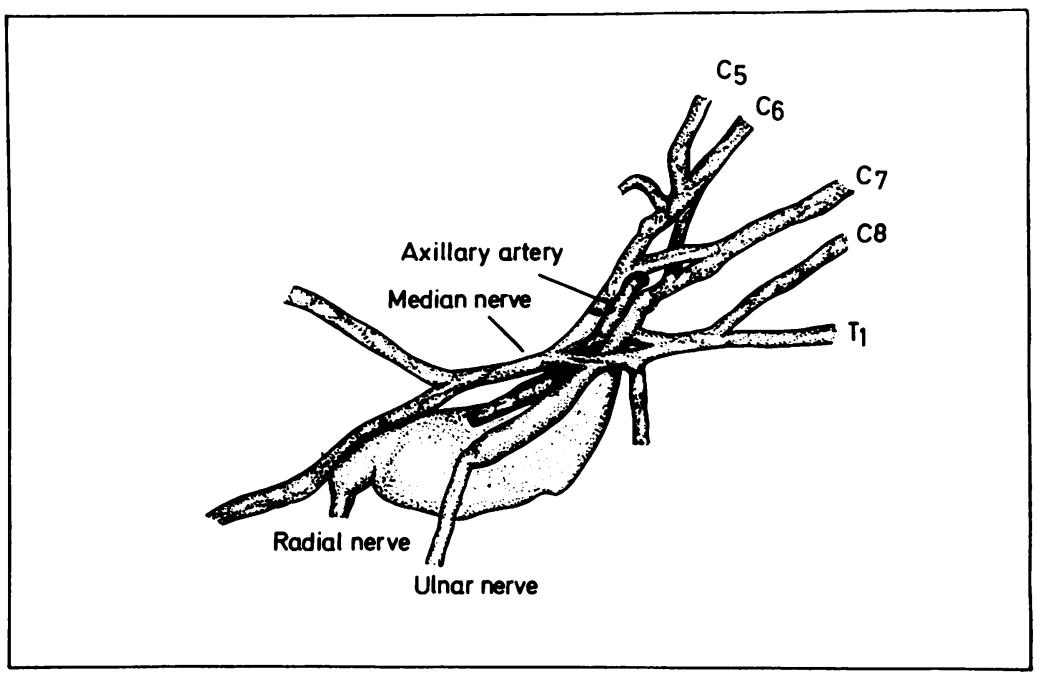

Fig. 1 Diagrammatic representation of the dissected necropsy specimen of the right brachial plexus showing fusiform expansion of the radial nerve by neurofibrosarcoma. Note the diffuse thickening of other nerves due to von Recklinghausen's disease.
Immediately next to the left adrenal gland there was a retroperitoneal haematoma containing an cstimated $750 \mathrm{ml}$ of clotted blood which appeared to have originated from a ruptured haemorrhagic adrenal neoplasm. Numerous grey nodules measuring up to $3 \mathrm{~mm}$ were seen both in the compressed remnant of this adrenal gland and in the adrenal gland on the other side.

The brain showed two haematomas measuring $50 \times 30 \times 40 \mathrm{~mm}$ in the centra ovalia of both frontal lobes. The surrounding parenchyma was oedematous and contained numerous punctate haemorrhages. Smaller haemorrhages measuring $10 \mathrm{~mm}$ were present in the frontal cortex, the corpus callosum, and the tuber cinereum.

Numerous somatic, sympathetic, and parasympathetic nerves and ganglia were grossly thickened, rubbery, and firm (Fig. 2), and cysts up to $3 \mathrm{~mm}$ in diameter containing gelatinous material were seen on section.

\section{MICROSCOPY}

Sections of biopsy and necropsy tissues from the right radial nerve mass showed an encapsulated tumour almost completely obliterating the architecture of the nerve. The tumour showed two distinct patterns of growth, one merging into the other. The first pattern, seen in the white whorled areas, consisted of spindle shaped cells arranged in a "herring-bone" pattern with numerous mitoses, many of them bizarre (Fig. 3). Thin walled vascular channels were present but the endothelial cells lining them did not have a malignant appearance. The intervening delicate fibrillary ground substance was alcian blue positive. These are the typical appearances of a neurofibrosarcoma (Harkin and Reed, 1969). The second histological pattern, seen in the haemorrhagic areas distant from the biopsy site, was that of an angiosarcoma. Malignant cells appeared to line a network of anastomosing vascular channels (Fig. 4). Reticulin stains of these areas emphasised the vascular architecture and

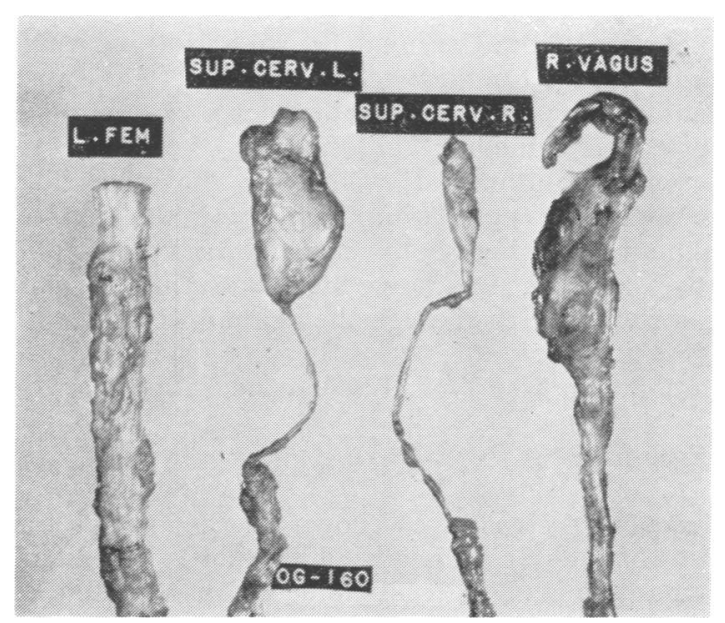

Fig. 2 Somatic and autonomic nerves at necropsy showing diffuse and focal thickening by von Recklinghausen's disease. From left to right: (1) left femoral nerve; (2) and (3) left and right superior cervical ganglia and sympathetic chains; (4) right vagus nerve. 


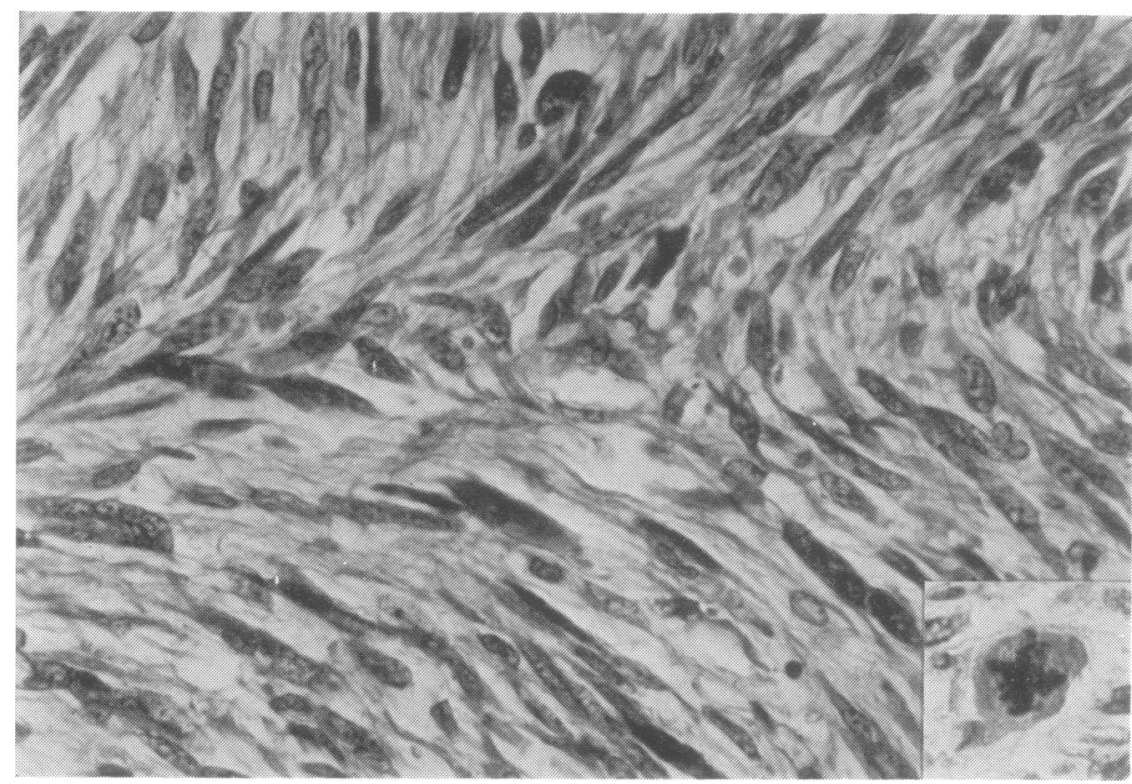

Fig. 3 Neurofibrosarcoma of right radial nerve showing hyperchromatic spindle shaped nuclei compactly arranged in herring-bone pattern. Inset: tumour cell with quadripolar mitotic figure. Both $H$ and $E \times 400$.

showed that the cells were inside the reticulin sheath of the vessels. In the transitional zones between these two patterns the cells became more anaplastic and lost their herring-bone arrangement with the formation of a few small blood vessels lined by malignant cells (Fig. 5).

Sections from the lung (Fig. 6) and brain metastases showed angiosarcoma in a pattern more distinct than that in the radial nerve. The adrenal cortex and medulla adjacent to the retroperitoneal haematoma, as well as on the other side, showed both nodular and diffuse infiltration by tumour showing both neurofibrosarcoma and angiosarcoma, the latter demonstrating a more compact pattern than in the primary lesion.

Sections of all the other peripheral nerve tumours showed typical benign neurofibromas.

\section{Discussion}

Vascular lesions are known to occur in von Recklinghausen's disease. They mostly consist of benign capillary haemangiomas, although Krucke (1974) has reported a case of generalised neurofibromatosis in the newborn in which haemangioblastoma and neurofibroma were closely intermingled in the cerebral pia mater, peripheral nerves and ganglia, skin, soft tissues, vertebral bodies, and several viscera.
In the case reported in this paper, the angiomatous tissue was obviously malignant, and its primary derivation in the radial nerve by metaplasia from the adjacent neurofibrosarcoma was established by the presence of transitional zones between the two.

Examples comparable with this case appear to be extremely rare, and have not so far been described in detail. Woodruff et al. (1973) quoted unpublished data on a case of angiosarcoma in a peripheral nerve tumour without giving details or illustrations. Another case is mentioned briefly, but not illustrated, by Russell and Rubinstein (1977). This was the case of a 35 year old male with classical von Recklinghausen's disease who had a neurofibrosarcoma with areas of chondroblastic metaplasia in the left femoral nerve. This had metastasised to the omentum and peritoneum where areas comprised of vascular spaces lined by malignant endothelium were closely associated with malignant neurofibrosarcoma. This case is described in more detail by Rubinstein (1963, case 2).

Divergent mesenchymal elements in peripheral nerve sheath tumours such as bone, cartilage, adipose tissue, and skeletal muscle may occur singly or in combination (Woodruff et al., 1973). This case illustrates another form of differentiation, and the presence of transitional 


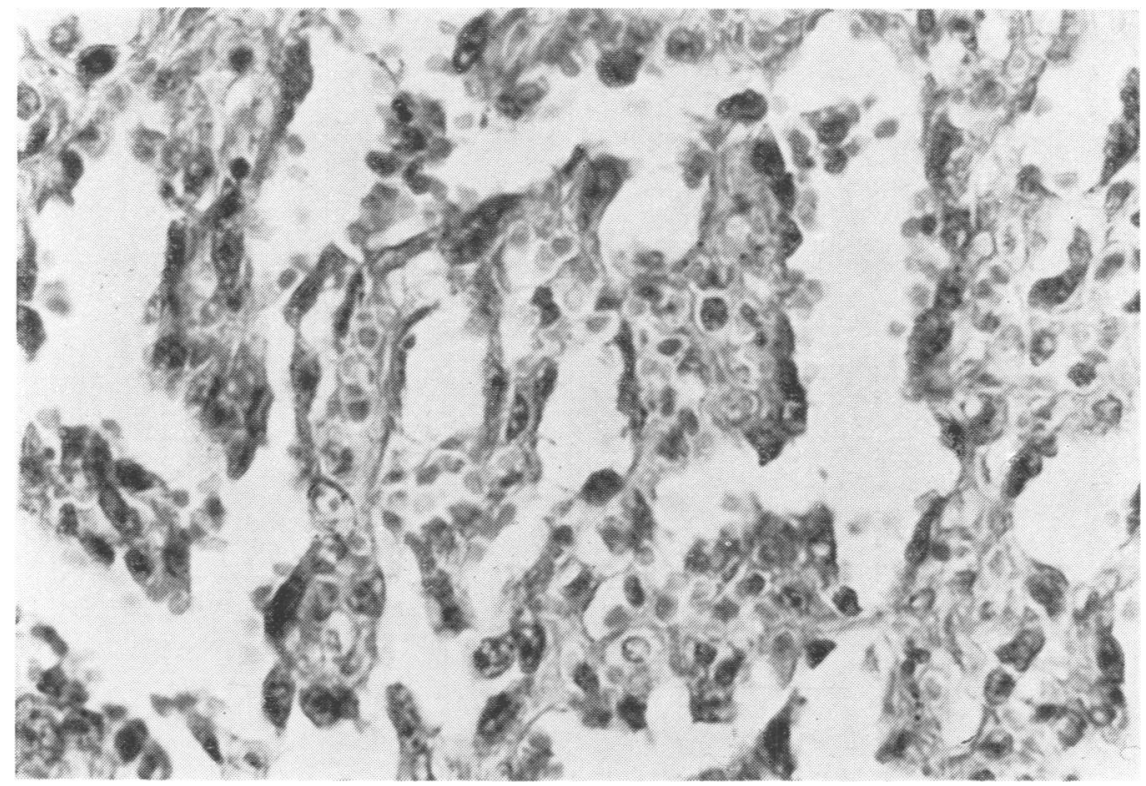

Fig. 4 Angiosarcoma in right radial nerve. Malignant cells line well-developed vascular spaces containing erythrocytes. $H$ and $E \times 400$.

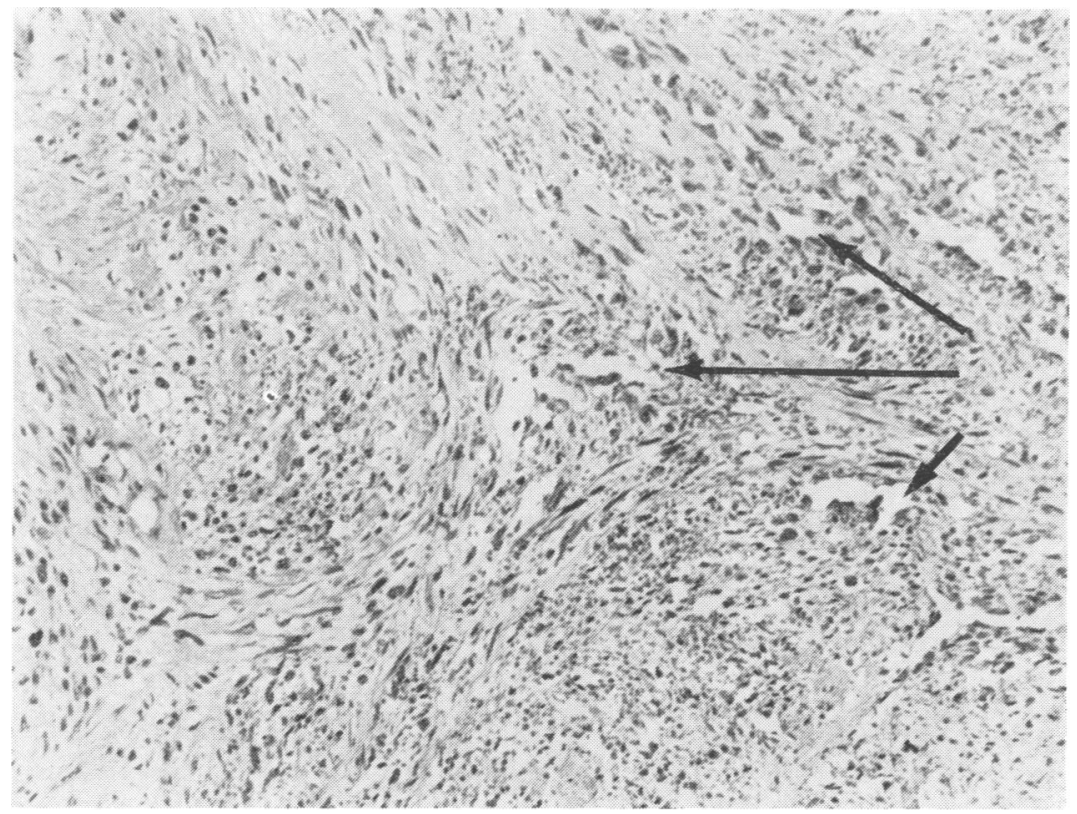

Fig. 5 Transitional zone between neurofibrosarcoma and angiosarcoma in right radial nerve. The cells are less compactly arranged than in Fig. 3 and are beginning to line ill-defined branching channels at centre and right (arrows). $H$ and $E \times 140$. 


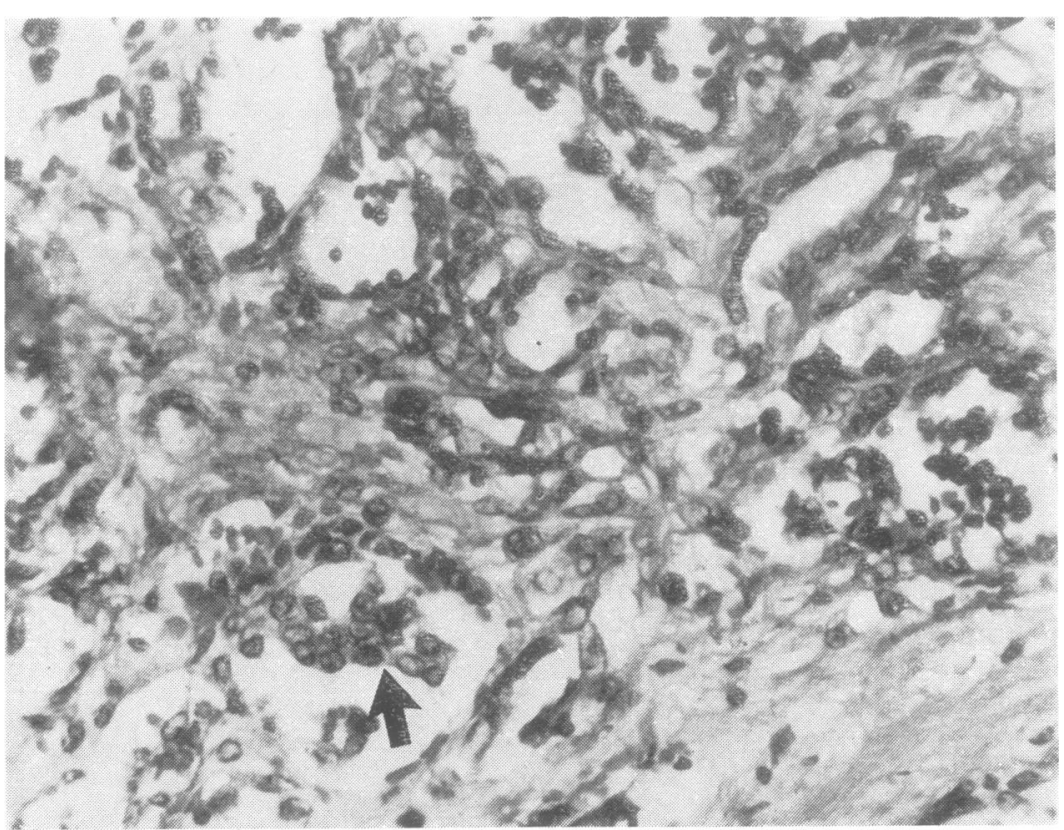

Fig. 6 Angiosarcomatous metastasis in right lung. Note the polypoid tuft of malignant cells growing into the lumen of the vessel at lower left (arrow). $H$ and $E \times 400$.

zones provides further evidence that all these variants represent metaplastic potential of malignant nerve sheath cells. Of special interest in this context is the simultaneous presence of divergent chondroblastic metaplasia in the only other example of angiosarcomatous differentiation with which the present case can be compared.

In two series (White, 1971; Woodruff et al., 1973), metaplastic elements made no significant difference to the prognosis. However, in this case, the haemorrhagic nature of the angiosarcomatous metastases in the brain rather than the actual extent of tumour growth undoubtedly caused the patient's death.

I would like to acknowledge the encouragement and criticism of Professor L. J. Rubinstein during the preparation of this paper. The work was supported in part by Graduate Neuropathology Training Grant 5 TO1 NS-05500-10 of the US Public Health Service (Dr L. J. Rubinstein).

\section{References}

Harkin, J. C., and Reed, R. J. (1969). Tumors of the peripheral nervous system. In Atlas of Tumor Pathology, Fasc. 3 (2nd series). Armed Forces Institute of Pathology: Washington DC.

Krucke, W. (1974). Pathologie der Peripheren Nerven. In Handbuch der Neurochirurgie. Edited by $\mathbf{H}$. Olivecrona, W. Tonnis and W. Krenkel. Volume 7, part 3. Springer-Verlag: Berlin.

Rubinstein, L. J. (1963). Tumeurs et hamartomes dans la neurofibromatose centrale. In Phacomatoses Cerebrales. Edited by L. Michaux and M. Feld, pp. 427-452. SPEI: Paris.

Russell, D. S., and Rubinstein, L. J. (1977). Pathology of Tumours of the Central Nervous System. Fourth edition. Edward Arnold: London.

White, H. R. (1971). Survival in malignant Schwannoma. Cancer, 27, 720-729.

Woodruff, J. M., Chernik, N. L., Smith, M. C., Millet, W. B., and Foote, F. W. (1973). Peripheral nerve tumours with rhabdomyosarcomatous differentiation (Malignant "Triton tumours"). Cancer, 321, 426-439. 